\title{
Extended Proxy Mobile IPv6 Scheme Using Global Local Mobility Anchor
}

\author{
Eshraga Hussien Elfadil \\ Department of Computer Networks, Faculty of Computer Science and Information Technology, University of Science \\ and Technology (UST), Khartoum, Sudan., \\ Email: eshragaalfadil@hotmail.com

\section{TajElsir Hassan Suliman} \\ Department of Information Technology, Sudan Academy for Banking and Financial Sciences (SABFS), Khartoum, \\ Sudan. \\ Email: tagsir@sabfs.edu.sd
}

\author{
Ahmed Hamza Osman \\ Department of Information System, Faculty of Computing and Information Technology, King Abdulaziz University \\ (KAU)-Rabigh, Saudi Arabia \\ Email: ahoahmad@kau.edu.sa
}

Received: 24 June 2021; Accepted: 03 August 2021; Published: 08 October 2021

\begin{abstract}
The internet was basically designed for the static nodes, but with the development of mobile nodes such as smart phones, that have wireless capabilities, the first design was insufficient. MNs change their point of attachment while they are roaming (traveling) in the internet, to maintain the survival of ongoing sessions for these mobile nodes, the internet requires techniques for managing mobility.. Currently, there are two types of mobility management protocols, host-based protocols and network-based protocols, the involvement of MN in the mobility process is must in the first type, while is unnecessary in the second type. The IETF standardized the Proxy Mobile IPv6 (PMIPv6) protocol in 2004, to overcome the limitations experienced by the host-based protocol, Mobile IPv6 (MIPv6) such as sub-optimal routing, handover latency, packet loss and single point of failure, however, the biggest drawback of PMIPv6 is the lack of inter-domain handover. This paper provides an efficient scheme based on standard PMIPv6 called (E-PMIPv6) to support inter-domain handover by introducing a new entity called (GLMA) which enables MN to traverse different domains while keeping the ongoing sessions, additionally; we use buffering techniques to preemptively lighten packet losses. The ultimate goal for the suggested scheme is to solve the scalability problem for the PMIPv6 and it is extensions to encourage network operators to deploy E-PMIPv6 for large networks. Results of preliminary analysis of handover latencies and related packet losses favored (E-PMIPv6) over two of the leading contenders.
\end{abstract}

Index Terms: PMIPv6, E-PMIPv6, FH-PMIPv6, HL

\section{Introduction}

The inter-technology handover process can be achieved through virtual interfaces in the PMIPv6 protocol (VI). In the Virtual Interface (VI), the Link Layer Identifier (LL-ID) is used to define the network interface and is used for the communication of the data link layer. The primary aim of the VI is to keep the physical interface a mystery (PI). The VI is designed to preserve the secret of the PI revolution by using the same LL-IDs if they are in the same technologies (e.g. IEEE 802.11). Unfortunately, only one interface can be supported, i.e. it cannot per time switch the address. The key concern facing VI preparation is substitute instructors, and this is described as the swapping dilemma. However, the PMIPv6 Flexible and multi-homing Protocol does not allow multi-homing because of Swapping problem. The PMIPv6 has a single Internal Gui. This various interactions make swapping complicated.

The Internet is a system for moving data packets. IPv4 is the very first version of the internet protocol [1]. The term "next-generation" is often referred to as "Internet Protocol next-generation" (IPng). This IPv4 IP supplies separate IP addresses for each computer device. However, IPv4 addresses have run out [2]. IPv6 is the latest IPv4. IPV6 implemented by Request for Comments (RFC) [3] It implements a protocol for host-based mobility management. The first mobility management protocol is Mobile IPv6, which was developed by the Internet Engineering Task Force (IETF). 
Depending on the protocol, the MN's presence during the signaling flow may be needed. In this mode, host-based Mobile IPv6 is required to retain the mobility information. In the new networking world, wireless communication is defined by the next-generation network (NGN). This NGN is learned with WiMAX, WLAN, Long Term Evolution (LTE) and 3GPP (3GPP). Proxy Mobile IPv6 (PMIPv6) is specified in IETF specifications. PMIPv6 is a network-based mobility control protocol that doesn't require the use of IEEE 802.1 LAN. In PMIPv6, the MAG transports the mobility linked signal alone without the involvement of the MN, however, the same role of MN is performed by the MAG in MIPv6. PMIPv6 uses only one tunnel to connect. However, the SVI of PMIPv6 is not adequate to sustain the NGN. The MN must be increased with several virtual interfaces to boost its networking reliability. The network-based accessibility management protocol PMIPv6 facilitates multi-homing via Mobile Virtual Interface. Signaled message flows begin with the assignment of specific Home Network Prefixes (HNP) per TSI [6]. The handover of signaling happens by way of VI. VI is concealing the advancement of the first layer i.e. physical interface from the upper layer i.e. logical interface. Instead of preserving VI, the secret of the session would be held. VI has two clauses that help layer recognition. Which is a mutual LL-ID and the other is an unshared LL-ID, in which case the former has a security issue because of the risk of spoofing devices' addresses. The second clause has an issue with an individual PI and VI. Often the neighbor was discovered and it was reported to have a molecular weight between MN and MAG. The MN can resolve the swapping problem by following the protected Neighbor Discovery protocol [10], which contains the encrypted LL-ID. But when the VI adjusts the security of LL-ID, then security will be collapsed. The trouble with the second clause. This paper details an effective inter-technology handover mechanism between IPv4 and IPv6 networks across a single tunnel. This can be attained by the use of Adversarial Surfing in PMIPv6.

Current mobile network, especially 3G, 4G and 5G employ a centralized mobility management, called PMIPv6, which is an enhanced version of standard mobility protocol MIPv6. Proxy Mobile IP (PMIPv6) overcomes the main drawback of the MIPv6 by performing mobility management functions utilizing a new network element called Mobility Access Gateway(MAG) that tackles the mobility functions on the sake of MN, hence eliminates the need for adding MIPv6 function to every mobile node on the network. In PMIPv6, the two communication parties(i.e CN and MN) can communicate with each other by using the Home of Address(HoA) of the MN without modifying the IP protocol stack, therefore, the mobile node can connect to a visited network by using the same IP address (HoA) it is assigned, and the CN only knows the MN's HoA, moreover, all designated packets are intercepted by a new network element called Local Mobility Anchor(LMA), which acts as a Network Address Translator(NAT) and manages the binding between the HoA and Care of Address(CoA) which assigned by the MAG for the MN while it is roaming, in addition LMA builds a tunnel with the MAG to send the corresponding packets designated to the MN. The idea of mobile management was pioneered with the advent of various wireless systems and the coexistence of their implementations of cellular networks, such as GSM, GPRS, and UMTS, all of which had WiFi network connectivity. Thus, a mobile node will roam freely between the various access networks. We have a lot of work to do and do in order to pave the way for an all-IP mobility combination of the Internet and telecommunication networks. Web IPv6 is the most prevalent work on the road to full mobile Internet access. In addition, the IETF's uniform protocol for mobility control, PMIPv6 [2] has drawn significant interest. The legacy Mobile IPv6 is an upgrade to Proxy Mobile IPv6 which offers network-based mobility control for legacy users. . Table 1 illustrates a comparison between MIPv6 and PMIPv6 from technical perspective.

Table 1.Comparsion between MIPv6 and PMIPv6

\begin{tabular}{|l|c|c|}
\hline \multicolumn{1}{|c|}{ Protocol Criteria } & MIPv6 & PMIPv6 \\
\hline Protocol Type & Host-based & $\checkmark$ \\
\hline MN managing multiple IP addresses & $\checkmark$ & Xetwork-based \\
\hline MN Protocol Modification & $\checkmark$ & $\checkmark$ \\
\hline Mitigating Packet overhead & $\checkmark$ & $\checkmark$ \\
\hline $\begin{array}{l}\text { Discovering the pre-configured IP address and Mobility } \\
\text { Anchor-MA }\end{array}$ & X \\
\hline $\begin{array}{l}\text { Large signaling overhead to maintain ongoing sessions } \\
\text { during handover }\end{array}$ & $\checkmark$ & $\checkmark$ \\
\hline Deployment by Network Operators & X & $\checkmark$ \\
\hline
\end{tabular}

Because of their variations, PMIPv6 should be used along with MIPv6. To give another example, Giaretta proposes using MIPv6 for worldwide mobility and PMIPv6 for local mobility The IETF's NETLMM workgroup suggests a mechanism for choosing an individual to carry the signaling: either within the $\mathrm{MN}$ or an access network. According to the draft, Han et al., a PMIPv6 indication and discovery mechanism [5], the changed prefix form in the Router Advertisement (RA) and Solicit messages may be used to hold the prefix. In addition, MIPv6 and PMIPv6 are under consideration for mobility control in 3GPP designing a system architectures [7] which are critical to 3G science. MIPv6 and PMIPv6 usage scenarios are investigated as well a subset of these studies deal with design concepts in order to pick a mobility management system, while the others examine potential deployment scenarios for optimized MIPv6.

For the remainder of this paper, the sections are structured as follows. In Section 2 we begin with a description related work of the MIPv6 and PMIPv6. In section 3, we present research scope and goals, in section 4 we present the architecture of the E-PMIPv6 and it is main operations (registration, handover) however, performance evaluation and 
results discussion is seen in section 5 by applying a mathematical model. The paper is concluded in section 6 by addressing the future works.

\section{Related Work}

Proxy Mobile IPv6 allowed mobility on top of Mobile IP to improve mobility management in MIPv6. The key variations between MIPv6 and PMIPv6 as well as other extensions have to do with the way the solution is applied. The network strategy makes services affordable to a vast number of consumers, which doesn't ask any service providers to make any improvements to how they provide services. Because MNs are not required to participate in mobility-related signaling, the network's overhead is minimized because the network controls mobility on the MN's behalf. As a network operator, adopting a network-based approach benefits them because it enables them to control the network's traffic and quality of service (QoS) through specialized networks. The existing multi-homing process within the network is depicted in Figure 1. PMIPv6's multi-homing capability enables a single router to support many interfaces. However, there are some circumstances in which concurrent "use" of several MN interfaces and the ability to move a single IP flow from one access technique to another require some enhancement/modification of the existing PMIPv6 base protocol [10]. Discusses the advantages and disadvantages of the fundamental concept of two PMIPv6 multi-homing models, including the use of a single unique prefix across all interfaces and a unique prefix per device. This article discusses the current Home Network Prefix (HNP) for all interfaces, as well as the mobility characteristics that the Home Network Prefix (HNP) offers in conjunction with micro-mobility features (HNP). If a router undergoes an interface transition, for example, the other interface may remain linked to the same router. To maintain communication across the network, the MN can swap out its WLAN access router for a $3 \mathrm{G}$ access router. When an MN is unable to connect to the network via the WLAN interface, a vertical handoff occurs at the 3G MAG in order to maintain session consistency via the cellular interface. However, in some instances of disconnection, the MN does not have enough time to initiate a vertical handoff at 3G MAG prior to packet loss. According to the PMIPv6 protocol, prefixes cannot be dynamically assigned to wired interfaces, and the $\mathrm{MN}$ is unable to relocate the prefix associated with a degraded interface contains a list of the many mobility management protocols[12].

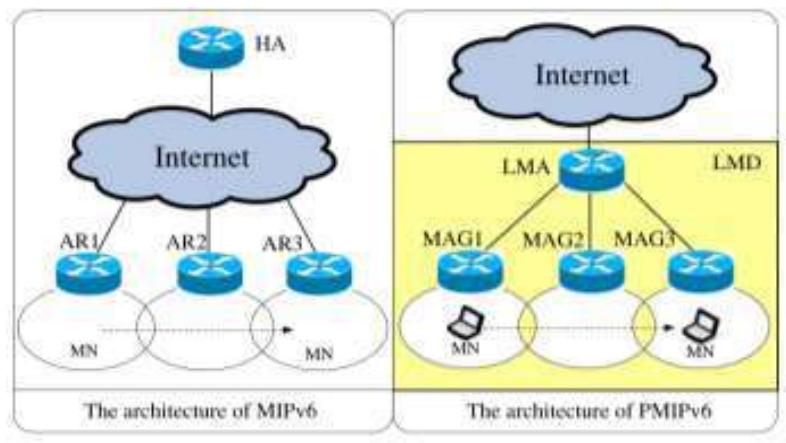

Fig. 1. The architectures of MIPv6 and PMIPv6

There are several ways to support multi-homing with IPv6. Different renewable registrations is discussed in $[12,14]$. To have multi-homing advantages, all mobile network prefixes were registered with the home network. Mobile routers used L2-tunnels and knowledge exchange to gain fault tolerance and selection of routes. The shim protocol offers advancement toward multi-homing. Here, the Shim Protocol was temporarily implemented in the Local Mobility Domain (LMD) to connect a Multi-homed MN to the LMA. A flow distribution scheme called a Shim mesh was proposed to provide a compact multi-homing technique. It was allowed by a Host Identity Protocol (HIP). It merges the current identifier/locator split architecture introduced by HIP with the micro-mobility management system of PMIPv6 that will be updated to support Global Mobility Management in the future. The LISP and shim6 techniques include techniques to facilitate multi-homing to the terminal system. The current bicasting technique led PMIPv6 to the firsthop destination. Here in this case, the LMA expanded its Binding Cache Entry (BCE) to fulfill the criteria for several bindings and an MN performs the binding update to LMA. The transmission of data packets by the LMA to the NewMobile Access Gateway (N-MAG) as well as the Previous-Mobile Access Gateway. (P-MAG). The same feature is provided by, at least, [19]. This was an improved multi-hop protocol which accounts for interface differences. This scheme offers a more versatile multi-homing support system and thus ensures consistency when transitioning from IPv6 to IPv4. In current multi-homing techniques, route discovery, shim protocol, and mobility control are employed, but not at the same time. The writing did not discuss the nuances of handoff and multiuser interfaces. This technique, [18], is just concerned with handing over. The solution [19] fits only for one-hop in multi-home systems. This piece of evidence does not support the drop off mechanism. In these previous studies, a flow mobility mechanism has been used to boost the efficiency of many heterogeneous networks. This suggested SAND framework improves performance of the PMIPv6 protocol based on handover and the various interfaces used in the multi-homing domain. 
Proxy Mobile IP (PMIPv6) provides network-based mobility for mobile nodes without needing them to engage in mobility. Despite its benefits, PMIPv6 still has a big drawback: Inter-domain handover is not allowed. The MN switches to a new PMIPv6 domain, which removes all ongoing sessions [1].

When an MN roams between MAGs in the PMIPv6 domain, a possible handover for FPMIPv6 (PMIPv6) includes a fast switch (proactive approach). FPMIPv6 enables the $\mathrm{CN}$ to reach an MN immediately upon its entry into the new network and subsequent attachment of the new MAG (nMAG). The previous MAG (pMAG) initiates the handover (HI) procedure, according to the Layer 2 information received from the MN. Prevent the MN from losing downlink packets during the tunnel transfer to the nMAG. Once the MN is connected, nMAG stores the packets and transmits them to the MN. The following description applies to FPMIPv6 signaling: When the RSS of an MN with the pMAG drops below the threshold, the MN begins scanning neighboring MAGs. The MAC layer determines the threshold. The MAC address and nMAG address of the MN are sent to the pMAG in Layer 2 reports. The pMAG creates a tunnel with the nMAG upon receipt of the report message in order to get information about the MN. After establishing the tunnel, the pMAG uses the tunnel to transmit MN packets to the nMAG. All packets coming are buffered by nMAG. When the MN joins the nMAG's network, it sends a Router Solicitation (RS) message. The nMAG flushes buffered packets to the $\mathrm{MN}$ after receiving the RS message from the MN. The nMAG interacts with the LMA in order to register the information about the MN and to create the tunnel between the nMAG and the LMA. Additionally, the LMA provides packets directly to the nMAG. The nMAG sends a RA message in response to the RS message [2].

\section{Research Scope and Goals}

\section{A. Enabling both intra-handover and inter-handover process for PMIPv6}

The ultimate goal of the proposed scheme is to enable both intra-handover (which is already supported in the standard protocol PMIPv6) and inter-handover (traveling among different domains) without dropping ongoing sessions.

Towards this direction, a new entity is added to the PMIPv6 architecture which is called (GLMA) located in between any two different domains and acts as Network Address Translator (NAT), and is in charge of supporting inter-handover process.

\section{B. Reducing Packet loss during inter-handover process}

The standard PMIPv6 suffers from packet loss during intra-handover process; however, we except there will be a great loss of packets during both intra and inter-handover process.

To end that, a buffering mechanism is applied to mitigate the packet loss to an ignorable rate.

\section{Research objective}

The goal of the research presented in this paper is to extend the standard network-based protocol (PMIPv6) to support both intra and inter handover process, to provide flexibility and scalability for the mobile networks. The ultimate goal is to solve the scalability problem of PMIPv6 and it is extensions by introducing a new entity called (GLMA) which maintains a dynamically updated binding cache as the mobile node traveled among different domains. As a result, the proposed scheme will encourage network operators to deploy the extended PMIPv6 (E-PMIPv6) for large networks.

As discussed in the related work section, the majority of proposed schemes focused on enhancing the standard PMIPv6 protocol which supports only intra-handover process for localized networks, hence limits it is deployment by the network operators.

Significant advancements have already been made in the development of schemes that capable of extending PMIPv6 to support inter-handover process.

The current research aims to contribute in two ways to this approach: a) Extending PMIPv6 to solve it is scalability problem, b) Reducing packet loss during both intra and inter-handover process. Our scheme is an enhancement of the schemes proposed in $[4,5]$.

\section{Proposed E-PMIPV6 architecture and Operations}

Fig. 2 depicts the proposed architecture. 


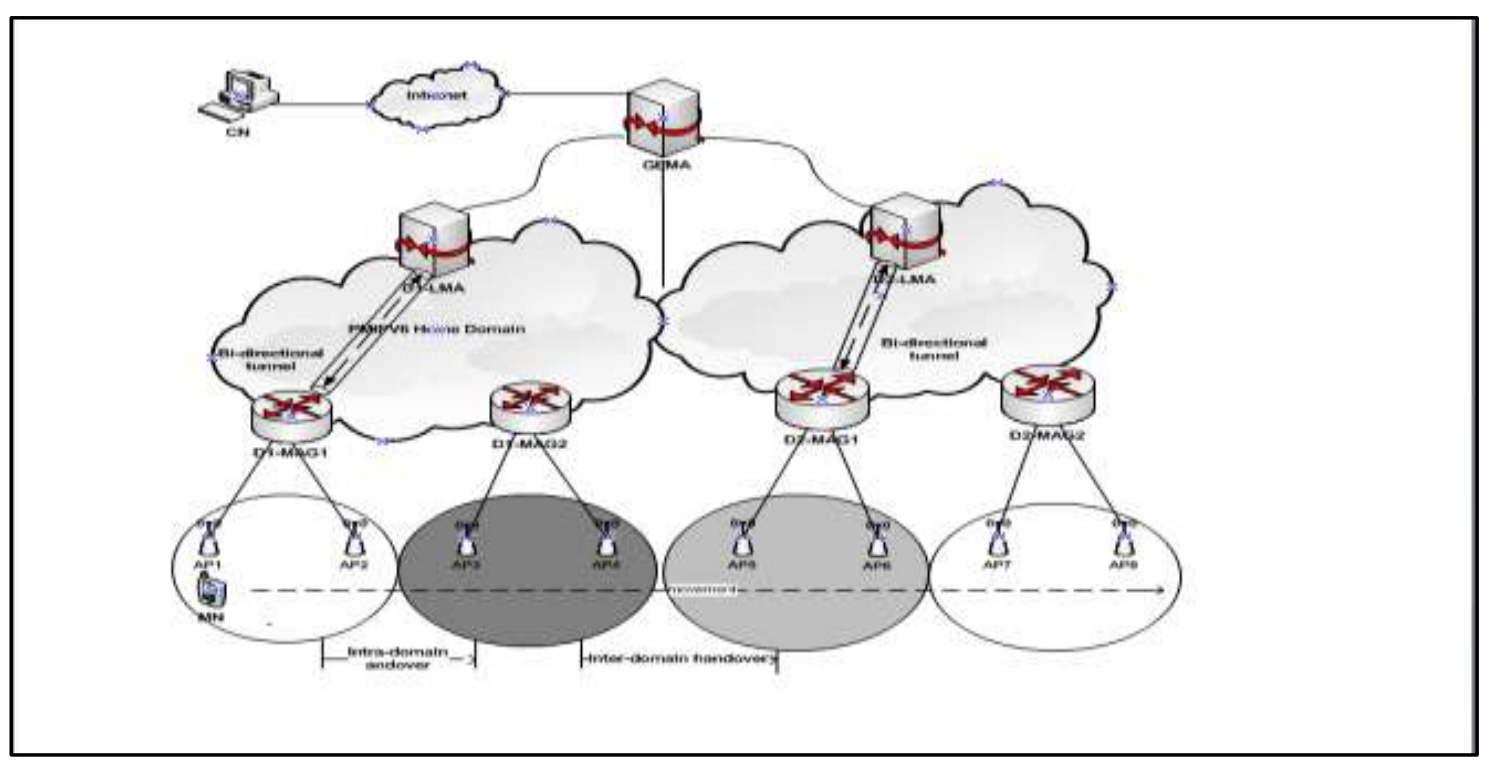

Fig.2. E-PMIPv6 Architecture

Fig. 2 illustrates the architecture of E-PMIPv6 and explains the entities that are used:

- Mobility access gateway (MAG): is an access router that manages a mobile node's mobility-related signaling. It is responsible for monitoring the mobile node's motions and signaling the mobile node's local mobility anchor.

- Mobile IPv6-domain: is a network that includes one link-layer address for a mobile node and multiple linklayer address for a MAG.

- Local Mobility Anchor: It is the organization that operates the mobile nodes, and all traffic from and to the mobile node is routed through the Local Mobility Anchor.

- Mobile Node: refers to a device which connects to the Learning Management Device wirelessly

- Corresponding Node $(\mathrm{CN})$ : Any node in the Internet or computer network that connects with an Electronic Medical Record (EMR).

- The Mobile Node's Home Address (MN-HoA) is the prefix for the mobile node's home network.

- Proxy Binding Update (PBU): is a message issued by a MAG to the LMA that establishes a connection between the MN's home network prefix (es) and its current care-of address (Proxy-CoA), and includes the fields MN-ID, Proxy-CoA, and handoff indicator (HI).

- Proxy Binding Acknowledgment (PBA): a return message received by a mobile entity to the entity that is the mobility anchor.

- $\quad$ Binding Cache (BC): Binding Cache held by the LMA that includes binding.

- Binding Cache Access (BCEs): Contains entries from the LMA's binding cache. And has fields for MN-ID, MAG Proxy-CoA and MN-prefix.

- GLMA is the body that is situated in the space between two domains and is responsible for liaising in between those two domains. This is achieved by doing a diligent intra-domain location registration prior to the $\mathrm{MN}$ traveling around various domains while roaming.

Fig. 3, 4, 5 and 6 demonstrate functions of the proposed architecture. A further discussion in this section is on two main operations: registration and handover. Each of these operations is illustrated on next.

\section{A. Registration Process}

Step1: the MAG senses MN in its access link and the MN attached to D1-MAG1.

Step2: the D1-MAG1 sends a PBU message to update MN current location.

Step3: D1-LMA creates BCE record contains MN-HNP (MN-ID) and MAG's address (D1-MAG1).

Step4: the D1-LMA setup one way tunnel and send PBack to D1-MAG1.

Step5: the D1-MAG creates a record for MN in its (BUL) contains the MN-HNP binds with D1-LMA and setup end point tunnel towards the D1-LMA1, in addition, D1-MAG1 send a (Rtradv) message to MN advertising MN-HNP.

Step6: finally, up on receiving the (Rtradv) message MN now is able to organize its interface and get it is HoA (MN-HoA), which uses to transmit data traffic sessions to its $\mathrm{CN}$. As shown in fig.3. 


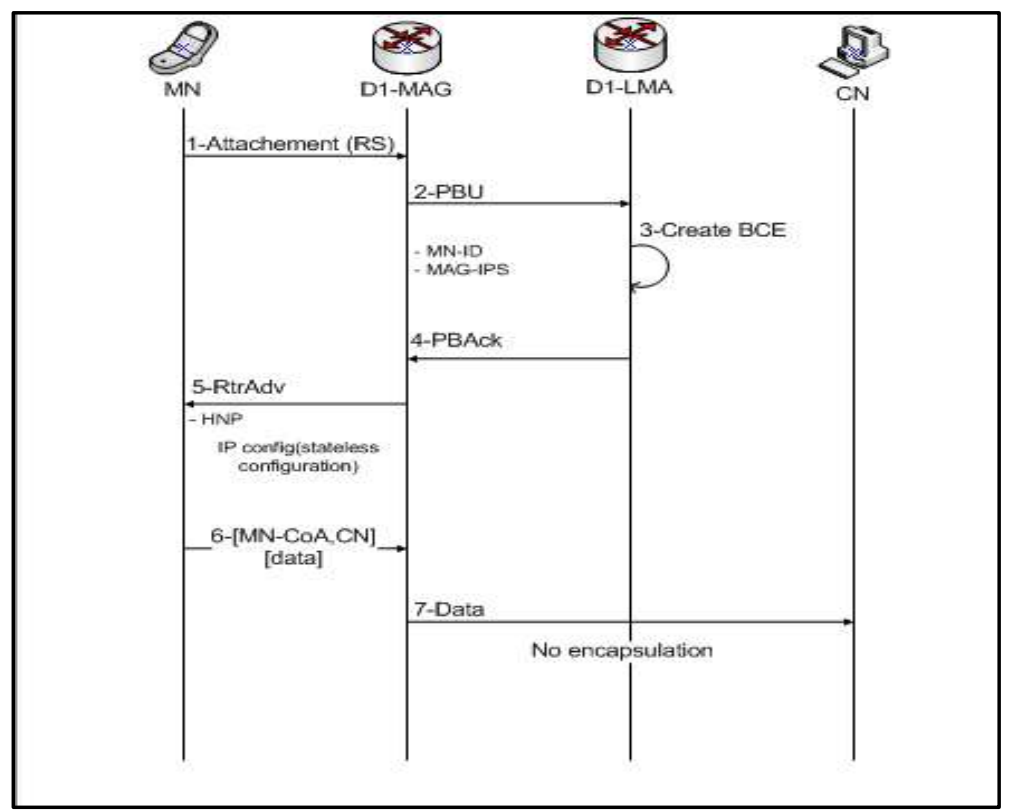

Fig.3. E-PMIPv6 Registration and Packet Delivery

\section{B. Intra Handover Procedure in E-PMIPv6}

MN still in D1-LMA, and the movement from D1-MAG1 towards the D1-MAG2 with in the same domain (D1).

Step1: whenever the D1-MAG1 detects MN intra handover to D1-MAG2 it will send a De-Reg PBU message to D1-LMA contains some information such as zero life time ( $\mathrm{t} 2)$, and no active session flag " $\mathrm{p}=0$ ".

Step2: the D1-LMA activates the pre-registration of MN actual detachment by sending a PBack message "with flag " $p=1$ ”. comprising of the MN profile towards the new D1-MAG2 neighbor based on the BCE list.

Step3: the D1-MAG2 must keep the MN information which received it from D1-LMA for a certain period of time, it will bypass the access authentication by comparing the MN "ID and HNP" with the pre-registered profile.

Step4: after MN successfully matched the D1-MAG2 updates MN BUL entry and sends RtrAdv message with same MN-HNP towards the MN, in order to reconfigure its interface to resume its data session.

Step5: D1-MAG2 sends PBU message to D1-LMA with flag $(\mathrm{P}=1)$ and new lifetime " $\mathrm{t} 2$ " as an indicator to the MN complete location update.

Step6: D1-LMA updates MN's BCE record with the new information; (D1-MAG2 ' HoA1.MN).

Step7: Finally, the D1-LMA directs the MN data traffic through the D1-MAG2 towards CN.

Fig. 4 depicts the intra-handover procedures.

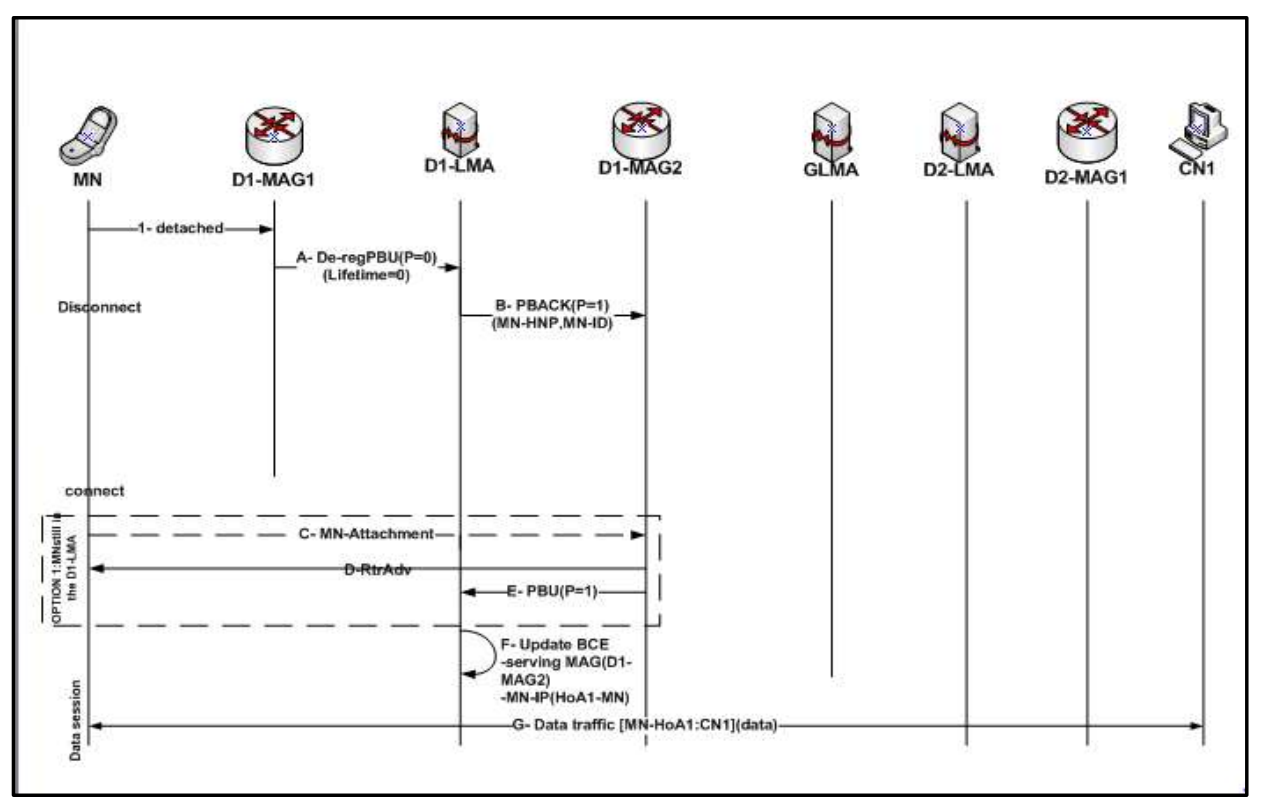

Fig.4. Intra-Handover Procedure in E-PMIPv6 
C. Inter Handover Procedure in E-PMIPv6

When MN moves to D2-LMA towards the D2-MAG's (e.g.: D2- MAG1, D2-MAG2) in this case the time that D1MAG2 is keeping information is over ( $\mathrm{t} 2>$ min delivery time).

In this case, there are two options:

Option1: in this option, if the session is not active, the details of steps are discussed as follows:

Step1: as soon as the D2-MAG1 senses the MN imminent to inter domain handover towards D2-MAG's (D2MAG1) access link, it sends a DeReg PBU message to D1-LMA with (zero life time and P="0").

Step2: D1-LMA sends EX.PBU to the GLMA that contains MN context and new life time ="t2".

Step 3\&4: once the D2-MAG1 detects MN attachment, it will perform the access authentication process by sending PBU to D2-LMA.

Step5: after authentication took place, D2-LMA sends EX-PBU message which contains context profile (MN-ID) to GLMA in order to authenticate the MN.

Step6: The GLMA activates the proactive inter-domain registration by sending EX-PBAck message comprising of MN profile towards the D2-LMA in order to update LCE.

Step7\&8: D2-LMA sends RtrAdv to D2-MAG1 which contains MN-HNP to confirm the MN context information and it is also sends PBU message towards D2-MAG1 to update the record.

Step9: moreover, the D2-LMA sends PBU message towards GLMA and it contains the new information of MN (MN-ID, D2-MAG1, IP, HNP2) to save inside the pre-registered information.

Step10: D2-MAG1 sends RtrAdv message to update it is record and to configure the new IP address (HoA2-MN). Step11\&12: MN sends data to CN1 using the configured IPv6 through D2-MAG1, and it sends this data directly to CN1 through D2-LMA and GLMA.

Fig. 5 illustrates the inter-handover procedures.

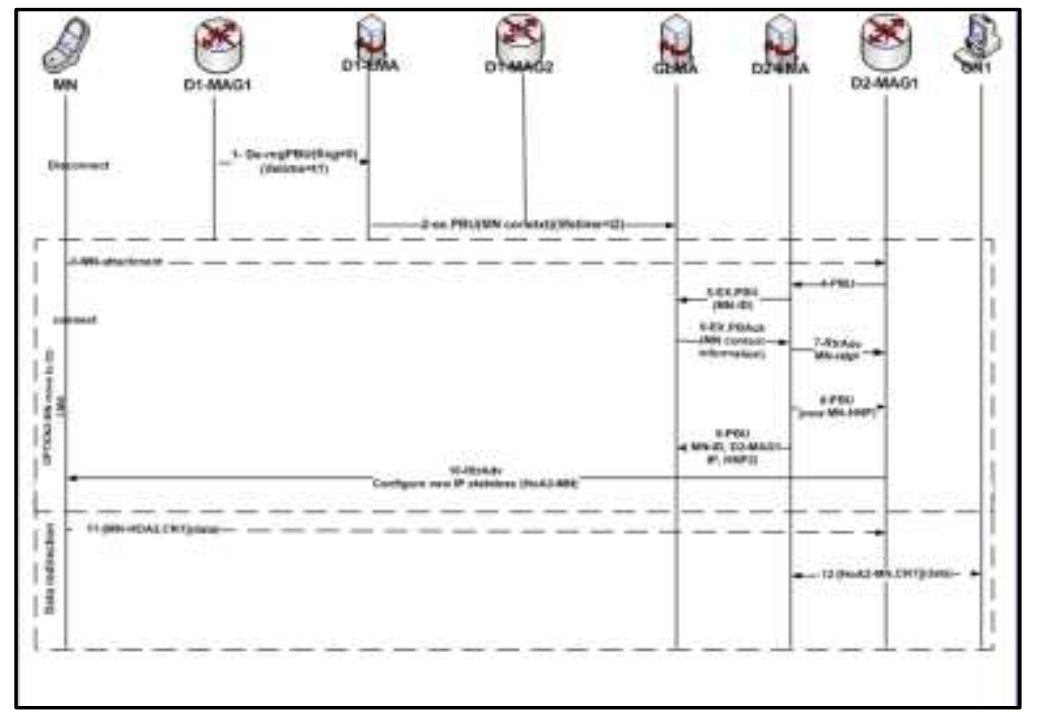

Fig.5. Inter Handover Procedure in E-PMIPv6 (without active session)

Option2: if the session is active, in this case the steps are discussed as followed:

Step1: whenever the D1-MAG1 senses the MN imminent for inter-domain handover towards the D2-MAG's (D2MAG1 OR D2-MAG2) access link within (zero life time and flag set to "1" as indicator to the active session).

Step2: in this step, the D1-LMA sends EX.PBU message to GLMA which contains the MN context and new life time " 2 ", as sequence.

The GLMA begins buffering packets bound for MN until to receive the BU message from another LMA to prevent the packet loss.

Step3.4: once the D2-MAG1 detect MN attachment it will perform the access authentication process by sending PBU to D2-LMA.

Step5: D2-LMA sends EX-PBU message which contains context profile (MN-ID) to GLMA in order to authenticate the MN

Step6: The GLMA activates the proactive inter-domain registration by sending EX-PBAck message comprising of MN profile towards the D2-LMA in order to update LCE. 
Step7\&8: D2-LMA sends RtrAdv to D2-MAG1 which contains MN-HNP to confirm the MN context information and it also sends PBU towards GLMA that contains the MN new information's (MN-ID, D2-MAG, IP, HNP2) to save the preregistered information.

Step9: D2-MAG1 sends RtrAdv message towards MN to configure the new IP address (HoA-MN) and to update it is record.

Step10: GLMA starts to forward the buffered data to MN through D2-LMA and D2-MAG1, respectively.

Step11: now MN can send the packets (HoA2-MN, data) to CN1 through D2-MAG1.

Step12: as sequence, D2-MAG1 sends the data to GLMA through D2-LMA and in this case the GLMA acts like NAT by changing the header from (HoA2-MN, data, to HoA1-MN, data) before forwarding this data to CN1.

Step13: finally, the GLMA forwards data directly to CN1.

Figure 6 depicts the inter-handover procedures with active session.

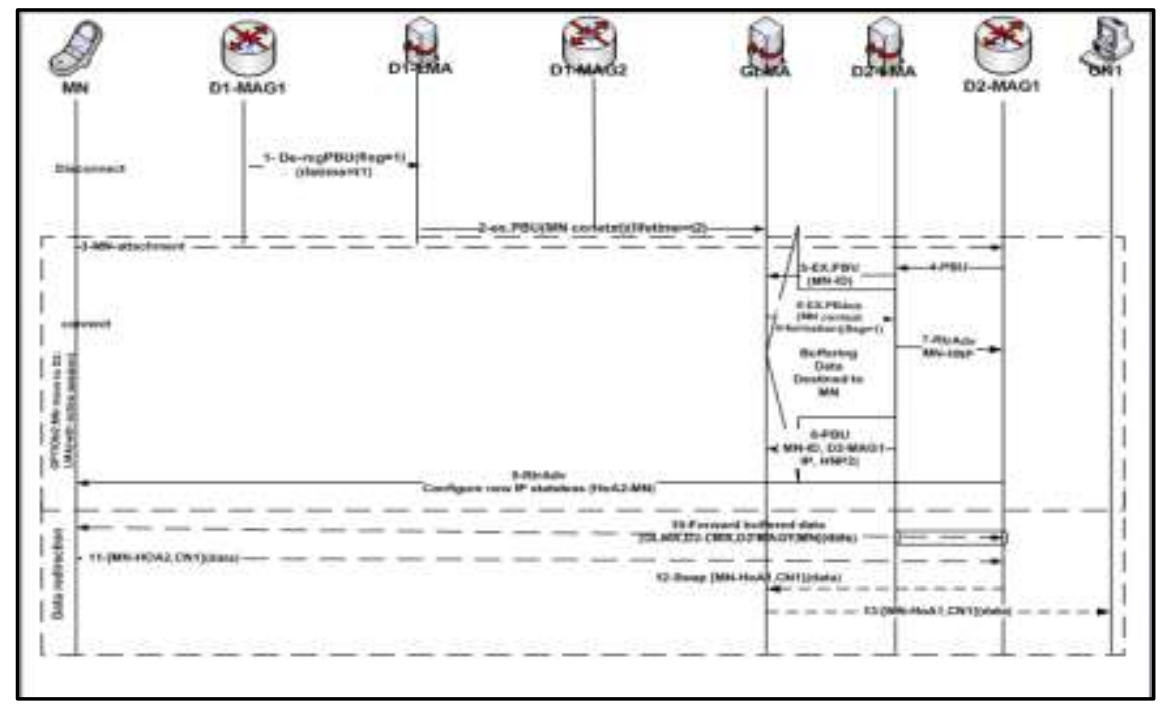

Fig.6. Inter-Handover Procedure in E-PMIPv6 (with active session)

\section{Performance Evaluation and Result Discussion}

To evaluate the effectiveness of the proposed E-PMIPv6, a mathematical model is used to measure three metrics as Handover latency(HL), Packet Loss(PL) and Signaling Overhead(SO), the obtained results are compared with two of the leading contenders, standard PMIPv6 and FH-PMIPv6, and the authors applied the same equations derived from [4] for aforementioned three schemes.

The evaluation process is described in details in the following sections, and table 2 depicts the obtained results by applying the mathematical model to measure the three metrics.

Table 2. A summary of obtained results for the three metrics (for three schemes)

\begin{tabular}{|c|c|c|c|c|c|c|}
\hline \multirow[t]{2}{*}{ Scheme } & \multicolumn{2}{|c|}{ Handover Latency(H.L)(seconds) } & \multicolumn{2}{|c|}{ Packet Loss(Bytes) } & \multicolumn{2}{|c|}{ Signaling Overhead(Bytes) } \\
\hline & Intra-Domain & Inter-Domain & Intra-Domain & Inter-Domain & Intra-Domain & Inter-Domain \\
\hline PMIPv6 & 0.121 & - & 3.368 & - & 152 & - \\
\hline FH-PMIPv6 & 0.109 & - & 3.039 & - & 228 & - \\
\hline E-PMIPv6 & 0.053 & $\begin{array}{l}0.060 \text { (inactive) session } \\
0.059 \text { (active)session }\end{array}$ & 0.000 & $\begin{array}{l}0.000 \text { (inactive) session } \\
0.000 \text { (active) session }\end{array}$ & 304 & $\begin{array}{l}\text { 456(active )session } \\
\text { 532(inactive) session }\end{array}$ \\
\hline
\end{tabular}

\section{A. Handover Latency (H.L)}

In our assessment, the Handover Latency HL takes on a crucial role. The HL is the time between the last time a MAG can communicate through the $\mathrm{MN}$ and the first time a MAG can communicate through the MN. Clinical Leukocyte Antigen, HL as follows:

$$
H L(.)=\mathrm{TL} 2+\mathrm{TMD}+\mathrm{TLU}
$$

Where TL2 denotes the Layer 2 (L2) handover latency and TMD denotes the movement detection latency in the following equation:

$$
\mathrm{TMD}=2 \mathrm{dwl}(\mathrm{M})
$$


Where $\mathrm{M}$ denotes the size in bytes of a control message, and TLU is the location update latency.

In PMIPv6, the new MAG obtains information about the MN's LMA via a DNS lookup. Then, using the LMA, the new MAG performs the PBU operation.

$$
\mathrm{T}_{\mathrm{LU}}(\text { pmipv6) }=2 \mathrm{dar}, \operatorname{dns}(\text { Mpmipv6) }+2 \mathrm{dar}, \operatorname{lma}(\text { Mpmipv6) }
$$

In FH-PMIPv6, the new MAG1 forwards the information to MAG2 and then performs the PBU process with the LMA and MAG2 simultaneously.

$$
\mathrm{T}_{\mathrm{LU}}(\mathrm{FH}-\mathrm{pmip})=2 \mathrm{dMAG} 1, \mathrm{MAG} 2(\mathrm{MFH}-\mathrm{mip})+2 \mathrm{dLMA,MAG} 2 \text { (MFH-pmip) }
$$

In our proposed scheme, E-PMIPv6, there are two options when the MN detached the domain, in option1 (intradomain handover) the previous D1-MAG1sends PBU to D1-LMA when it senses MN imminent from intra-domain handover towards D1-MAG2.then, D1-LMA sends PBAck to D1-MAG2 and receives PBU from D1-MAG.

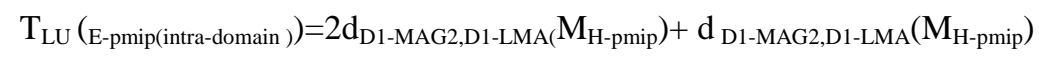

In option 2 (inter-domain handover) we assumed two options:

Firstly: if the session is not active: in this case D1-MAG1 sends PBU to D1-LMA where senses MN imminent inter-domain handover and then performs the PBU and PBAck process among D1-LMA, GLMA, D2-LMA, D2-MAG1 simultaneously.

$$
\begin{gathered}
\mathrm{T}_{\mathrm{LU}}(\mathrm{E} \text {-pmip(inter-domain(without active session })=3 \mathrm{~d}_{\text {D2-GLMA,D2-LMA }}\left(\mathrm{M}_{\mathrm{E}-\mathrm{pmip}}\right)+2 \mathrm{~d}_{\text {D2-MAG,D2-LMA }}\left(\mathrm{M}_{\mathrm{E}-\mathrm{pmip}}\right)+ \\
\mathrm{d}_{\text {D1-MAG1,D1-LMA }}\left(\mathrm{M}_{\mathrm{E}-\mathrm{pmip}}\right)+\mathrm{d}_{\text {D1-LMA,GLMA }}\left(\mathrm{M}_{\mathrm{E}-\mathrm{pmip}}\right)
\end{gathered}
$$

Secondly: if the session is active: in this case D1-MAG1 sends PBU to D1-LMA where senses MN imminent inter domain handover with activate session and then performs PBU and PBAck process a mong D1-LMA, GLMA,D2LMA,D2-MAG1, moreover in this case GLMA starts buffering exchanged data and saves them until it destined to MN.

$$
\begin{aligned}
& \mathrm{T}_{\mathrm{LU}}\left(\mathrm{E}_{\text {-pmip(inter-domain(with active session })}\right)=3 \mathrm{~d}_{\mathrm{D} 2-\mathrm{GLMA}, \mathrm{D} 2-\mathrm{LMA}}\left(\mathrm{M}_{\mathrm{E}-\mathrm{pmip}}\right)+\mathrm{d}_{\mathrm{D} 2-\mathrm{MAG} 1, \mathrm{D} 2-\mathrm{LMA}}\left(\mathrm{M}_{\mathrm{E}-\text { pmip }}\right)+ \\
& \mathrm{d}_{\text {D1-MAG1,D1-LMA }}\left(\mathrm{M}_{\mathrm{E}-\mathrm{pmip}}\right)+\mathrm{d}_{\text {D1-LMA,GLMA }}\left(\mathrm{M}_{\mathrm{E}-\mathrm{pmip}}\right)
\end{aligned}
$$

Fig. 7 illustrates the handover latency in seconds for the three schemes with $0.121 \mathrm{~s}, 0.109 \mathrm{~s}$ and $0.053 \mathrm{~s}$ for intradomain and 0.060 for inter-domain without active session and $0.059 \mathrm{~s}$ for inter domain with active session, respectively.

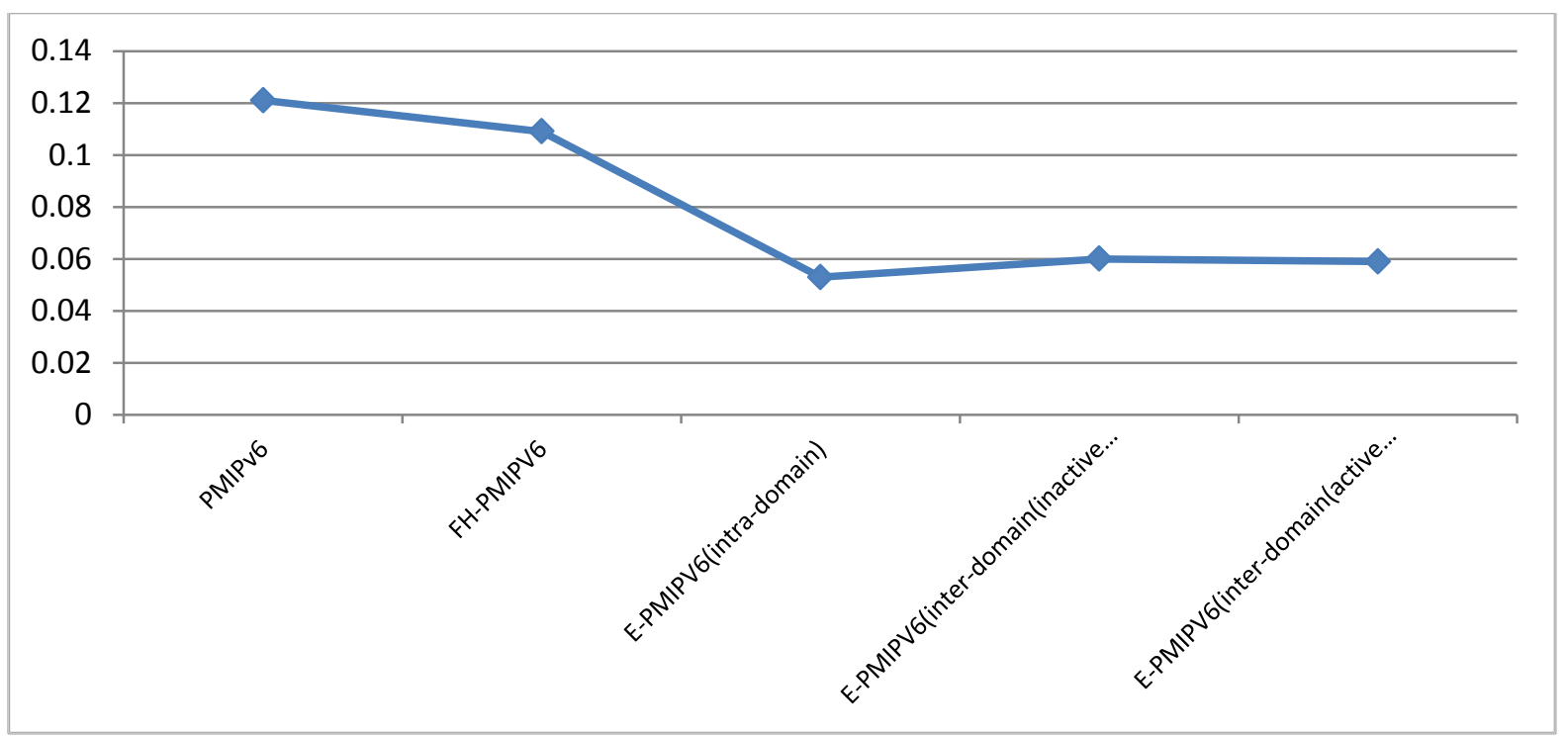

Fig.7. Handover Latency for PMIPv6, FH-PMIPV6 and E-PMIPv6 (intra/inter-domain) handovers

After applying equation (1), which is calculated from the equations (2), (3), (4), (5), (6) and (7) respectively, it appears that the E-PMIPv6 scheme significantly reduces the HL compared to other counterpart protocols. 


\section{B. Packet Loss (PL)}

The packet loss is a measure of how many packets are lost from a given network after a handover. Packet loss is inversely proportional to both the delay of handover and the number of packets that arrive at the destination. The packet arrival rate for the three schemes can be calculated as the product $\lambda \mathrm{s} \mathrm{Sp}$.

PL is generally expressed as follows [4] :

$$
\operatorname{PL}(.)=\lambda s \mathrm{Sp} \mathrm{HL}(.)
$$

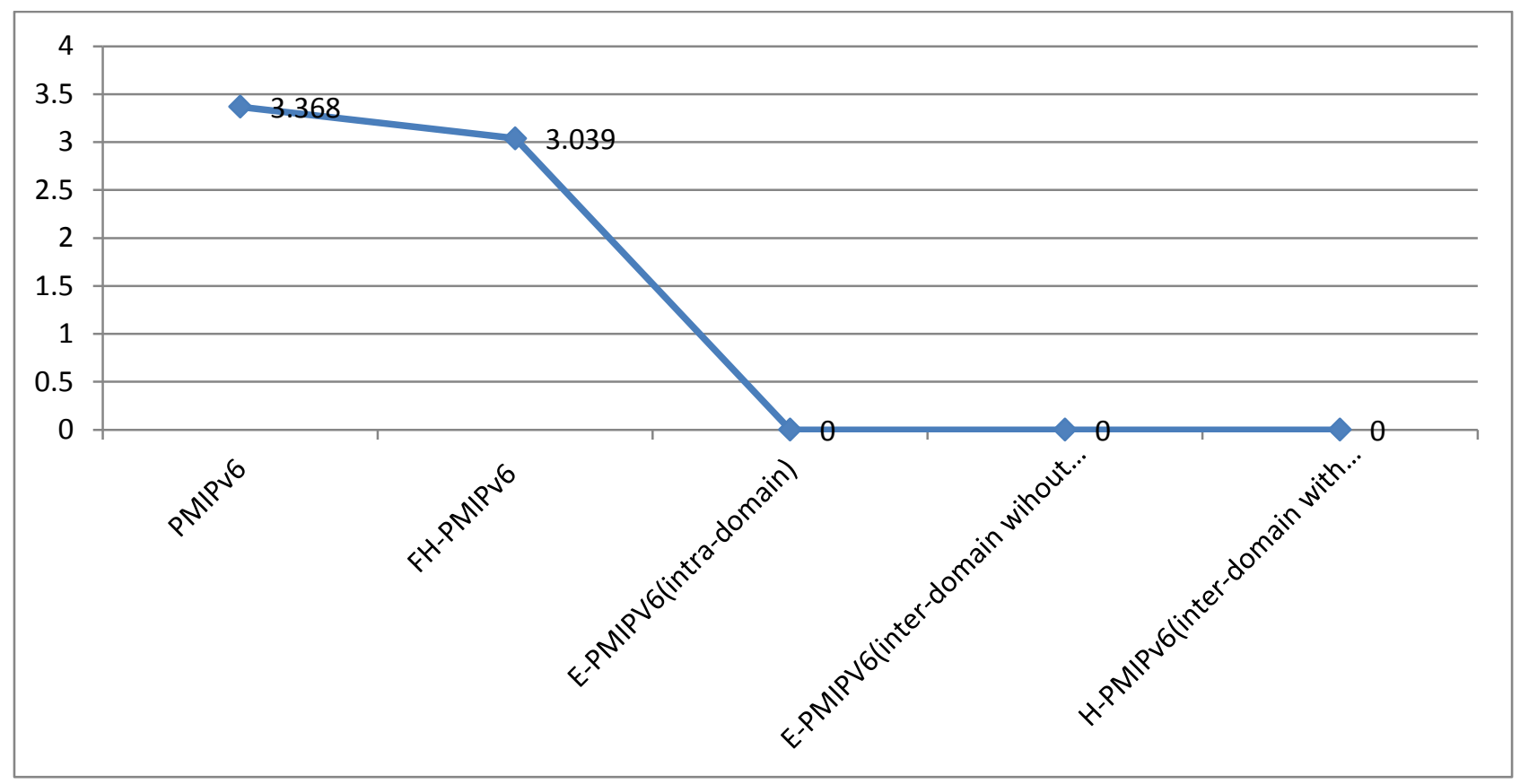

Fig.8. Packet loss of PMIPv6 and FH-PMIPv6 and E-PMIPv6 (intra/inter-domain handover without and with active session)

Fig.8 illustrates packet loss for PMIPv6, FH-PMIPv6 and E-PMIPv6 (intra/inter domain handover without and with active session) (3.368), (3.039) and (0.000) respectively.

After applying equation (8) for the three schemes, we noticed, that PMIPv6 scheme has the largest PL value which is proportional to HL, therefore the PL in FH-PMIPv6 is less than PMIPv6, and in contrast E-PMIPv6 uses buffering mechanism at the GLMA as a solution for packet loss. Such solutions become more viable and flexible with CMM technology as each anchor recognizes all of its targets simultaneously.

\section{Signaling Overhead (SO)}

Signaling overhead (SO) means the total number of control messages sent over the network links during the handover procedure, multiplied by the size of these messages in bytes.

For PMIPv6, the signaling overhead is:

PBU message sent from the nMAG to the LMA, plus PBA message received by then MAG.

$$
\mathrm{SO}=\operatorname{Mpmipv6}(\mathrm{PBU}+\mathrm{PBA})
$$

For FH- PMIPv6, the signaling overhead is: MAG2.

PBU+De-Reg PBU message sent from the MAG2 to the LMA; plus PBA+De-Reg PBA message received by the

$$
\mathrm{SO}=\mathrm{M}_{\mathrm{FH}-\mathrm{pmip}}(\mathrm{PBU}+\mathrm{De}-\mathrm{Reg} \mathrm{PBU}+\mathrm{PBA}+\mathrm{De}-\mathrm{Reg} \mathrm{PBA})
$$

For E-PMIPv6, the signaling overhead is:

First: in option1 (intra-domain handover) PBU message is sent from D1-MAG1 to the D1-LMA; plus EX-PBU message receives by D1-LMA, plus PBAck message from D1-MG2 to D1-LMA.

$$
\mathrm{SO}=\mathrm{M}_{\mathrm{E}-\mathrm{pmip}(\text { intra-domain) }}(\mathrm{PBU}+\mathrm{PBA}+\mathrm{EPBU})
$$


Second: in option2 (inter-domain handover) if the session is not activate (without active session): in this case PBU message is sent from D1-MAG1 to D1-LMA, plus EX-PBU message from D1-LMA to GLMA, plus PBU from D2MAG1 to D2-LMA and EX-PBU message from D2-LMA to GLMA, plus received PBAck message from GLMA, plus PBU message from D2-LMA TO GLMA and D2-MAG1, respectively.

$$
\mathrm{SO}=\mathrm{M}_{\mathrm{E}-\mathrm{pmip} \text { (inter-domain(without active session) }}(\mathrm{PBU}+\mathrm{PBU}+\mathrm{PBU}+\mathrm{PBU}+\mathrm{PBU}+\mathrm{EXPBA}+\mathrm{EX}-\mathrm{PBU})
$$

If the session is activate(with active session):in this case PBU message sent from D1-MAG1 to D1-LMA, plus EXPBU message from 1-LMA to GLMA, plus PBU from D2-MAG1 to D2-LMA, plus EX-PBU message from D1-LMA to GLMA, plus received PBAck message from GLMA, plus PBU message from D2-LMA TO GLMA .

$$
\mathrm{SO}=\mathrm{M}_{\mathrm{E}-\mathrm{pmip}(\text { inter-domain(with active session) }}(\mathrm{PBU}+\mathrm{PBU}+\mathrm{PB}+\mathrm{EX}-\mathrm{PBA}+\mathrm{EX}-\mathrm{PBU}+\mathrm{EX}-\mathrm{PBU})
$$

Fig.9 illustrates the signaling overhead in bytes for the three schemes, PMIPv6 has the lowest signaling overhead (152 bytes), FH-PMIPv6 has the signaling overhead (304 bytes) and the E-PMIPv6 (intra/inter-domain handover) has highest signaling overhead (228 bytes for intra-domain handover) and (456 bytes for inter-domain handover (with active session) and (532 bytes for inter-domain handover without active session) respectively.

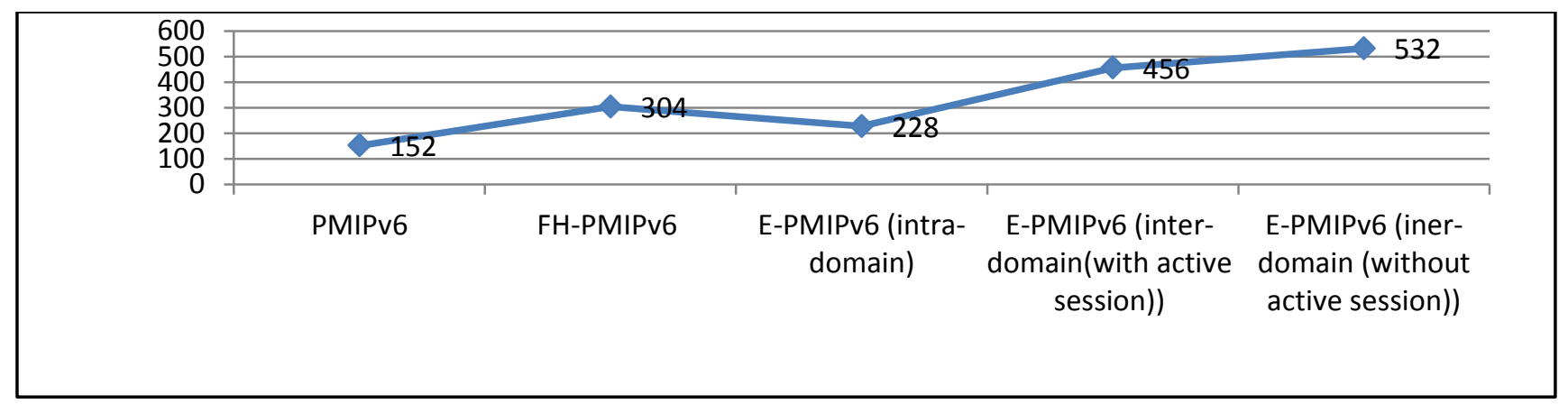

Fig.9. Signaling Overhead in PMIPv6, FH-PMIPv6 and E-PMIPv6

After applying equations (9), (10), (11), (12) and (13) respectively, for the three schemes, Unfortunately, both of FH-PMIPv6 and E-PMIPv6 cause much higher overhead (304 bytes, 228 bytes) respectively, in the network compared with PMIPv6 which has (152 bytes) and is due to the Information Retrieval request IR [18], PBU message sent to LMA and PBA as replay for MAG2 PBU message with 76 bytes as control message size as well as ( 228 bytes) in EPMIPv6 (intra-domain handover) due to PBU, PBA and EX-PBU from MN to previous MAG as illustrated before, and (456 and 532 bytes) in E-PMIPV6 (inter-domain handover with and without active session) due to 5 PBUs, EX-PBA, EX-PBU between the entities increased the overhead.

In the above results we ignored the RS and RA messages, since they are remain the same in the three schemes. Table 3 illustrates the formulas used for quantitative analysis.

\begin{tabular}{|c|c|c|c|}
\hline Symbol & Equation & Default values & Meaning \\
\hline $\begin{array}{l}\text { Wired link delay } \\
d_{\mathrm{x}, \mathrm{y}}(\mathrm{p})\end{array}$ & $d_{\mathrm{x}, \mathrm{y}}(\mathrm{p})=h_{\mathrm{x}, \mathrm{y}}\left(\mathrm{p} / \mathrm{B}_{\mathrm{wd}}+\mathrm{L}_{\mathrm{wd}}\right)$ & $\begin{array}{c}\mathrm{h}_{\mathrm{ar}, \mathrm{ar}}=\sqrt{\mathrm{N}} \approx 6, \mathrm{~h}_{\mathrm{ar}, \mathrm{db}}=\mathrm{h}_{\mathrm{ar}, \mathrm{lma}}=\mathrm{h}_{\mathrm{ar}, \mathrm{dns}}= \\
12 \text { hops }, \mathrm{h}_{\mathrm{mag}, \mathrm{mdb}}=\mathrm{d}_{\mathrm{mn}, \mathrm{mag}}=1 \text { hop } \\
\mathrm{P}=2 \text { bytes } \\
\mathrm{B}_{\mathrm{wd}}=100 \mathrm{Mbps} \\
\mathrm{L}_{\mathrm{wd}}=0.5 \mathrm{~ms}\end{array}$ & $\begin{array}{c}\text { h: the number of hops between } x, y . \\
\text { P: packet size in bytes. } \\
B_{w d} \text { : Bandwidth of wired link. } \\
\mathrm{L}_{\mathrm{wd}} \text { : propagation latency in wired link. }\end{array}$ \\
\hline Wireless link delay $\mathrm{d}_{\mathrm{wl}}(\mathrm{p})$ & $\mathrm{d}_{\mathrm{wl}}(\mathrm{p})=\left(\mathrm{p} / \mathrm{B}_{\mathrm{wl}}+\mathrm{L}_{\mathrm{wl}}\right) /(1-\mathrm{P} f)$ & $\begin{array}{c}\mathrm{B}_{\mathrm{wl}}=10 \mathrm{Mbps} \\
\mathrm{L}_{\mathrm{wl}}=2 \mathrm{~ms} \\
\mathrm{P} f=0.35\end{array}$ & $\begin{array}{c}\mathrm{B}_{\mathrm{wl}} \text { : bandwidth of wireless link. } \\
\mathrm{L}_{\mathrm{wl}}: \text { propagation latency in wireless link. } \\
\mathrm{p} f \text { : frailer probability of wireless link. }\end{array}$ \\
\hline Handover latency HL (.) & $\mathrm{HL}()=.\mathrm{T}_{\mathrm{L} 2}+\mathrm{T}_{\mathrm{MD}}+\mathrm{T}_{\mathrm{LU}}()$. & $\mathrm{T}_{\mathrm{L} 2}=10 \mathrm{~ms}$ & $\mathrm{~T}_{\mathrm{L} 2}$ : layer 2 handover latency. \\
\hline $\begin{array}{c}\text { Movement detection time } \\
\mathrm{T}_{\mathrm{MD}} \\
\end{array}$ & $\mathrm{T}_{\mathrm{MD}}=2 \mathrm{~d}_{\mathrm{wl}}(\mathrm{M})$ & $\mathrm{M}_{\text {pmipv6 }}=76$ bytes & $\mathrm{M}_{\text {pmipv6 }}$ : size of control message in bytes. \\
\hline $\begin{array}{c}\text { Location update latency } \\
\mathrm{T}_{\mathrm{LU}} \\
\end{array}$ & \multicolumn{3}{|c|}{$\mathrm{T}_{\mathrm{LU}}($ pmipv6 $)=2 \mathrm{~d}_{\mathrm{ar}, \mathrm{dns}}\left(\mathrm{M}_{\text {pmipv6 }}\right)+2 \mathrm{~d}_{\mathrm{ar}, \text { Ima }}\left(\mathrm{M}_{\mathrm{pmipv6}}\right)$} \\
\hline Packet loss PL (.) & $\operatorname{PL}()=.\lambda_{\mathrm{s}} \operatorname{Sp~HL~(.)~}$ & $\begin{array}{c}h_{\mathrm{s}}=2{ }_{\mathrm{s}} \\
\mathrm{Sp}=50 \text { packets }\end{array}$ & $\begin{array}{c}\lambda_{\mathrm{s}}: \text { session arrival mean rate. } \\
\text { Sp: average size of session in packets. }\end{array}$ \\
\hline Signaling overhead SO & \multicolumn{3}{|c|}{$\mathrm{SO}=\mathrm{M}_{\text {pmipv6}}($ number of control messages $)$} \\
\hline
\end{tabular}

Table.3. Formulas used for quantitative analysis

Source: Ref[5] 


\section{Conclusion}

This paper attempts to address the limitations experiences by the standard PMIPv6 protocol that doesn't support the inter-domain handover that limits it is deployment for large networks. An enhanced scheme is proposed called (EPMIPv6) to overcome the aforementioned problem, and mathematical analysis is used to evaluate the effectiveness of the proposed scheme in contrast with the standard PMIPv6 and FH-PMIPv6 in terms of handover latency, packet loss and signaling overhead.

The ultimate goal for the proposed scheme is to solve the scalability problem for the PMIPv6 and it is extensions to encourage network operators to deploy E-PMIPv6 for large networks

Obtained results of preliminary analysis of handover latencies and related packet losses favored the proposed scheme E-PMIPv6 over two of the leading contenders.

As part of future work, we plan to design and evaluate the performance of the proposed scheme using OMnet++ simulation software and enhancing the security of the scheme, since it was out of the this paper.

\section{References}

[1] http://datatracker.ietf.org/doc/search/?name=PMIPV6

[2] Kang B, Anh KQ, Choo H," Implementation of fast handover for proxy mobile IPv6: Resolving out-of-order packets”, PLoS ONE 12(10): e0182375. https://doi.org/10.1371/journal.pone.0182375, 2017.

[3] Fabio Giust, Carlos J. Bernardos, Antonio de la Oliva, " Combining client and network-based Distributed Mobility Management. " Telecommunication Systems, 2013

[4] Petro P. Ernest, Olabisi E. Falowo, H. Anthony Chan."Network-based Distributed Mobility Management." IEEE 9 ${ }^{\text {th }}$ International Conference on Wireless and Mobile Computing, Networking and Communications (WiMob),2013.

[5] Ibrahim Al-Surmi1, Mohamed Othman1,BorhanuddinMohd Ali2. "Hybrid Intra/Inter-domain Handover Mechanism for Superior Performance Enhancement Within/Across IP-Based Wireless PMIPv6 Domains Network".Springer Science+Business Media New York 2016.

[6] Wei Siang Hoh, SashikumarMuthut, Bi-Lynn Ong, Mohamed Elshaikh, MohdNazriMohdWarip and R. Badlishah Ahmad.“A SURVEY OF MOBILITY MANAGEMENT PROTOCOLS".VOL. 10, NO. 19, OCTOBER 2015.

[7] Hassan Ali-Ahmad, MeryemOuzzif, Xavier Lagrange. " Distributed Mobility Management: Approaches and Analysis." Communications Workshops (ICC), 2013 IEEE International Conference, Budapest, Hungary, 2013.

[8] Muhammad Zubair, Xiangwei Kong, SaeedMahfooz, and IrumJamshed, " SIDP: A Secure Inter-Domain Distributed PMIPv6. " International Journal of Information and Electronics Engineering, Vol. 4, No.2, Mar.2014.

[9] Hassan Ali-Ahmad, MeryemOuzzif, Xavier Lagrange. " Distributed Mobility Management: Approaches and Analysis." Communications Workshops (ICC), 2013 IEEE International Conference, Budapest, Hungary, 2013.

[10] Zhiwei Yan, and Guanggang Geng, Sherali Zeadally, and Yong-Jin Park."Distributed All-IP Mobility Management Architecture Supported by the NDN Overlay".IEEE, 2016.

[11] Fabio Giust, Antonio De La Oliva, Carlos J. Bernardos, Rui Pedro Ferreira Da Costa, "PMIPv6 A Network-Based Localized Mobility Management Solution.” The Internet Protocol Journal, Volume 13, No.3. Visit on 18 Feb 2017

[12] S. Gundavelli, K. Leung, V. Devarapalli, K. Chowdhury, B. Patil, RFC 5213 - Proxy Mobile IPv6, IETF Std., June 2015

[13] Ming-Chin Chuang and Jeng-Farn Lee. "FH-PMIPv6: A Fast Handoff Scheme in Proxy Mobile IPv6 Networks". Department of Computer Science and Information Engineering National Chung Cheng University, Chia-Yi, Taiwan, R.O.C.2013.

[14] Petro P. Ernest, Olabisi E. Falowo, H. Anthony Chan."Network-based Distributed Mobility Management." IEEE 9th International Conference on Wireless and Mobile Computing, Networking and Communications (WiMob),2013.

[15] Haritha G. "Distributed Mobility Management over IP based Mobile Networks." International Journal of Engineering and Technical Research (IJETR) ISSN: 2321-0869, Volume-3, Issue-2, Feb 2015.

[16] Kyoungjae Sun, Younghan Kim."Flow Mobility Management in PMIPv6-based DMM (Distributed Mobility Management) Networks." 2014.

[17] Muhammad Zubair, Xiangwei Kong, Saeed Mahfooz, and Irum Jamshed. "SIDP: A Secure Inter-Domain Distributed PMIPv6". International Journal of Information and Electronics Engineering, Vol. 4, No. 2, March 2014.

[18] Linoh A. Magagula1 and H. Anthony Chan. "IEEE802.21 OPTIMIZED HANDOVER DELAY FOR PROXY MOBILE IPV6".2013.

[19] Wei Siang Hoh, Sashikumar Muthut, Bi-Lynn Ong, Mohamed Elshaikh, Mohd Nazri Mohd Warip and R. Badlishah Ahmad. “A SURVEY OF MOBILITY MANAGEMENT PROTOCOLS”. VOL. 10, NO. 19, OCTOBER 2015.

[20] Ibrahim Al-Surmi1 • Mohamed Othman1 • Borhanuddin Mohd Ali2. "Hybrid Intra/Inter-domain Handover Mechanism for Superior Performance Enhancement Within/Across IP-Based Wireless PMIPv6 Domains Network". Springer Science+Business Media New York 2016.

[21] Farouk Abdul Jalin, Raed Alsaqour.“A Simulation Study Of Proxy Mobile IPv6 (PMIPV6) Protocol.” ARPN Journal of Engineering and Applied Sciences, Volume. 11, NO. 7, Apr 2016.

[22] TajElsir H. Suliman, Altayeb S. Abuelyaman, “ Distributed Mobility Management Scheme using Lightweight Fast Handover (DMM-LFH)", International Journal of Scientific \& Engineering Research(IJSER), Volume 4, Issue 10, October2013.

[23] Madeeha Aman, Saeed Mahfooz, Muhammad Zubair, Neelam Mukhtar, Kanwal Imran, Shah Khusro, "Tunnel-Free Distributed Mobiltiy Management(DMM) Support Protocol for Future Mobile Networks", Journal of Electronics Volume, 8, NO.1519, Dec,2019. 


\section{Authors' Profiles}

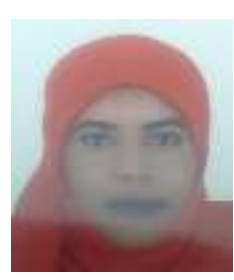

Eshraga Alfadil Hussain obtained her B.Sc in Information Technology from university of Garden City( Sudan), in 2010 and MSc. in Distributed Mobility Management (DMM) from university of Science and Technology( Sudan) in 2017, her main research interest include, DMM, wireless networks, proxy management, she has 7 years teaching experience in different fields of information technology, currently she is working as head of IT department at Omdurman Ahlyia university(Sudan).

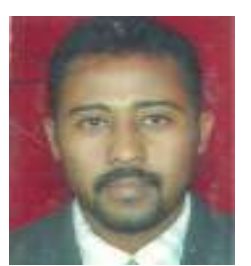

Dr.TajElsir Hassan Suliman obtained his BSc. in computer science from Sudan university of Science and Technology (Sudan) in 2000, and M. Sc. in computer science from school of mathematics, university of Khartoum (Sudan) in 2006, and PhD. In computer science in Distributed Mobility Management (DMM) from Sudan university of Science and Technology (Sudan) in 2016, he has more than 10 publications in international journals, and over 20 years teaching experience. Currently, he is working as an IT program coordinator at Sudan Academy for Banking and Financial Sciences (Sudan), his main research interests include, DMM, information security, wireless network, cloud security and AI.

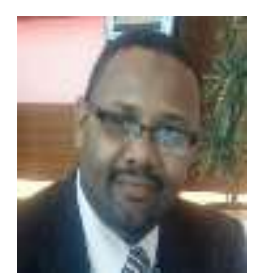

Assoc. Prof. Dr. Ahmed Hamza Osman graduated with a Bachelor of Computer Science from International University of Africa. He obtained his Master's Degree in Computer Science from Sudan University of Science and Technology, Sudan and his PhD in Computer Science with excellent academic achievements from Universiti Teknologi Malaysia (UTM). He was the Head of Computer Science department at the Faculty of Computer Studies at international University of Africa. Currently he works as Associate Professor in King Abduaziz University (KAU) Saudi Arabia.

How to cite this paper: Eshraga Hussien Elfadil, TajElsir Hassan Suliman, Ahmed Hamza Osman, "Extended Proxy Mobile IPv6 Scheme Using Global Local Mobility Anchor", International Journal of Information Engineering and Electronic Business(IJIEEB), Vol.13, No.5, pp. 38-50, 2021. DOI: 10.5815/ijieeb.2021.05.04 